\title{
Review
}

\section{Cognition in Huntington's Disease in Manifest, Premanifest and Converting Gene Carriers Over Ten Years}

\author{
Ellen P. Hart ${ }^{\mathrm{a}, *}$, Eve M. Dumas ${ }^{\mathrm{a}}$, Erik J. Giltay ${ }^{\mathrm{b}}$, Huub A.M. Middelkoop ${ }^{\mathrm{a}, \mathrm{c}}$ and Raymund A.C. Roos ${ }^{\mathrm{a}}$ \\ ${ }^{a}$ Leiden University Medical Center, Department of Neurology, Leiden, The Netherlands \\ ${ }^{\mathrm{b}}$ Leiden University Medical Center, Department of Psychiatry, Leiden, The Netherlands \\ ${ }^{\mathrm{c}}$ Leiden University, Institute of Psychology, Clinical Neuropsychology Unit, Leiden, The Netherlands
}

\begin{abstract}
.
Background: Cognitive decline in Huntington's disease (HD) remains an area of inconsistencies, especially far from disease onset.

Objective: To clarify the course of cognition in premanifest HD.

Methods: Twenty-six premanifest HD, 19 manifest HD, and 87 control subjects were followed for ten years, using an extensive cognitive battery. Differences in baseline levels and change over time, on four factors (motor speed, global cognition, executive functioning (EF), and memory) were examined, using multilevel regression analyses. Converters were additionally analysed as a separate group. Also, the influence of motor speed and predicted years to disease onset on the cognitive factors was studied. Results: Manifest HD subjects showed lower baseline scores compared to controls on the motor speed $(p=0.002)$, memory $(p<0.001)$ and EF $(p<0.001)$. They additionally deteriorated over the ten-year follow-up on memory $(p=0.01)$. Converters deteriorated on EF $(p=0.04)$. Further analyses of premanifest subjects 'far from and close to predicted onset' revealed lower baseline scores for the 'close' group on EF, as compared to controls $(p=0.001)$. They also deteriorated on memory $(p=0.01)$. Motor speed substantially mediated the results of the three cognitive factors; when added as covariate to the model several baseline and slope differences for the cognitive factors ceased to be significant.

Conclusions: Memory and EF are highly sensitive for ascertaining deterioration in premanifest HD gene carriers, especially in subjects close to onset. Lack of deterioration for the subjects further away from onset suggests that both domains are largely unaffected in those far from onset. Also, motor influence on cognition is substantial and should be taken into account in cognitive HD research.
\end{abstract}

Keywords: Huntington's disease, executive functioning, longitudinal, converters, premanifest

\section{INTRODUCTION}

Huntington's disease (HD) is an autosomal dominant neurodegenerative disease causing selective neuronal damage resulting in motor abnormalities, psychiatric disturbances and cognitive decline [1]. Due

*Correspondence to: Ellen P. Hart, MSc, Department of Neurology (J3-R-162), Leiden University Medical Centre, P.O. Box 9600, 2300 RC Leiden, The Netherlands. Tel.: +31 71 526 5442/2125; Fax: +31 71526 4466; E-mail: epthart@lumc.nl. to its genetic basis subjects at risk for HD can be tested for presence of the HD gene [2]. Consequently, gene carriers without a clinical diagnosis of disease onset, so-called premanifest carriers, can be identified and studied. In recent years the transition from premanifest to manifest HD has become a main area of interest. As we move forward towards clinical trials with possible disease modifying effects it is important to better understand the course of HD symptoms, including that of cognition. 
Since the localization of the HD gene [3] a large number of studies on cognition in premanifest HD have been published. Many cross-sectional studies have reported differences between premanifest and control subjects, with defective emotion recognition and reduced psychomotor speed being the most robust findings [4-6]. Concerning attention, memory and executive functioning inconclusive findings have been reported. For example, Verny et al. [7] found premanifest deficits on executive functioning and memory tasks compared to controls, whereas Witjes-Ané et al. [8] and Soliveri et al. [9] did not report such differences. In subjects close to their predicted age of disease onset, memory and executive deficits have been quite consistently found. In individuals further from predicted onset results are less robust, although psychomotor speed reduction and impaired emotion recognition have been observed [10, 11].

Few studies have assessed cognitive functioning in HD during follow-up periods of more than five years [12-15], despite the potentially informative nature of longer longitudinal studies, given the slow progressive nature of HD. These studies showed large differences in the kind and extent of the included cognitive batteries, duration of follow-up and number of participants included. Moreover, results are heterogeneous. Snowden et al. [12] found that colour naming on the Stroop task declines, Jurgens et al. [15] reported diminished concentration on a subtest of the Wechsler Memory Scale, in contrast to Brandt et al. [13] who found no cognitive decline over time.

As the development and progression of cognitive impairment associated with premanifest HD remains an area of uncertainty, specifically in the period far from disease onset, longitudinal research with substantial follow-up periods and comprehensive test batteries is important to increase our understanding of cognitive functioning in this critical period in HD. We assessed cognitive functioning at five time points during ten years of follow-up, allowing us to model the course of cognitive functioning and to compare the decline of (pre)manifest HD subjects with that of healthy controls. During the follow-up period we expect the HD groups to decline on tasks of memory and executive functioning compared to controls. For further analyses the premanifest group will be divided in those who converted to manifest disease over the study period, and those who remained premanifest. Furthermore, we will investigate the difference in cognitive change between premanifest HD subjects far from predicted disease onset and those close to onset as compared to controls, as we hypothesized that the predicted duration until disease onset at baseline is inversely associated with the rate of decline on certain cognitive scores. The effect of motor functioning on cognition will also be investigated.

\section{MATERIALS AND METHODS}

\section{Subjects}

At baseline 134 subjects participated (46 genecarriers, 88 controls) [8] (Fig. 1), 106 completed three-year follow-up [16] and 72 participants completed seven-year follow-up [15]. Ten-year follow-up was completed by 68 participants ( 26 gene carriers, 42 controls).

In this study we investigated all subjects over tenyear follow-up. Gene carriers were grouped at baseline into either premanifest HD gene carriers or manifest HD patients using the total motor score (TMS) of the Unified Huntington's Disease Rating Scale (UHDRS)

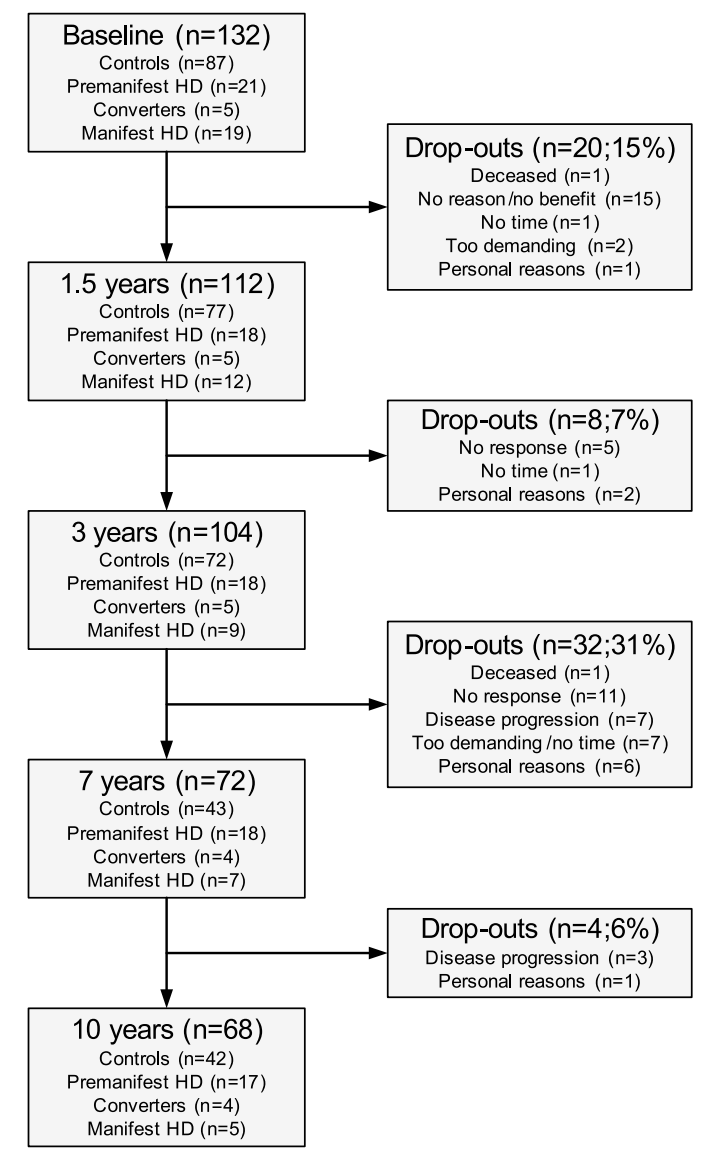

Fig. 1. Flowchart of the participants and drop-outs over 10 years of follow-up. 
[17]: subjects with scores below 6 points were considered to be premanifest, scores of 6 and higher were considered manifest [18]. Control subjects were tested non gene-carrying relatives.

Drop-outs over the ten-year period are described in Fig. 1. Of the 134 subjects one female gene-carrier was excluded from analyses as the TMS was not ascertained at baseline, precluding a correct assignment to one of the HD groups. One male control was excluded as he suffered from cardiovascular disease and consequent cognitive dysfunction. The remaining 132 subjects were included and grouped as follows: 19 manifest HD, 26 premanifest HD (of whom 5 converted to manifest HD over the study period), and 87 control subjects.

Predicted years to disease onset were estimated using the formula by Langbehn et al. [19]. The formula (age*(CAG repeat length-35.5)) was used to calculate disease burden for the HD groups. The 'sadness' score of the UHDRS behavioural section was used as depression measure. The study was approved by the local ethics committee and all participants gave written informed consent according to the Declaration of Helsinki.

\section{Procedure}

Following the baseline visit subjects attended follow-up visits after 18 months, three, seven and tenyears. During baseline, three and ten-year follow-up visits an extended cognitive battery was administered. For 18 months and seven-year follow-up a shorter battery was applied.

All participants underwent UHDRS motor, behavioural and functional assessments at all visits. The motor part was administered by a trained clinician. The cognitive battery included tests objectifying almost all cognitive domains. For global functioning the Mini Mental State Examination (MMSE) and the Wechsler Adult Intelligence Scale revised (WAIS-R) were used, the latter generating a total intelligence quotient (TIQ), performance (PIQ) and verbal (VIQ) intelligence quotients. Memory tests included the Wechsler Memory Scale (WMS) and the Dutch version of the California Verbal Learning test, namely the "Verbale Leer- en Geheugen test (VLGT)". From the WMS a memory quotient (MQ) can be derived. The VLGT consists of a main list to be learned (list A) and directly reproduced, and an interference condition (list B). Subjects are tested on direct recall, short term recall, long term recall and long term recognition. The Boston Naming (BNT) task was used for measuring picture naming abilities. For executive functioning the
Stroop test (colour, word and interference conditions), the written Symbol Digit Modalities test (SDMT), letter fluency ('f', 'a', 's'), and Trail Making test (TMT) (A and B) were used. A reaction time (RT) test, where participants were instructed to react as quickly as possible by releasing a 'rest' button and pressing a reaction button after hearing a tone (condition 1, 14 times) and seeing a light (condition 2, 14 times), was administered resulting in a RT score, from now on referred to as 'motor speed'. For detailed description of study procedures see Witjes-Ané et al. [8].

\section{Statistical analyses}

IBM SPSS for Windows 20.0 was used for data analysis. Group comparisons for demographic variables were done using ANOVA, $\chi^{2}$ and Kruskal-Wallis tests for continuous, categorical and skewed data, respectively. Data pre-processing involved reverse coding for TMT A\&B and RT, and logarithm ( $\log _{\mathrm{e}}$ ) transformation of non-normally distributed scores (i.e. TMT $\mathrm{A} \& \mathrm{~B}, \mathrm{MMSE}, \mathrm{BNT}$, and the short- and long-term recall and recognition subtests of the VLGT). For longitudinal analyses all cognitive scores were standardized to $z$-scores. These $z$-scores were subsequently combined into factors by averaging the component test results.

To make the data on the extensive cognitive battery more comprehensible and to reduce Type I error caused by multiple testing (19 individual cognitive tests were administered) we decided to perform a factor analysis to combine inter-correlated cognitive tests into factors. Based on results from previous cognitive studies in $\mathrm{HD}$, we were interested in the progression of performance on one motor construct and three cognitive constructs (global cognitive functioning, memory, executive functioning) in our cohort. The tests that are known, according to existing cognitive HD research, to capture these constructs were pre-selected to be entered in the factor analysis. The correlation coefficients of the variables had to be substantially large (correlation coefficient $\geq 0.30$ ) to be assigned to a specific factor. Principal components analysis (using varimax orthogonal rotation) revealed four factors: (1) "motor speed" (i.e., RT $z$-score) (2) "global cognition" (composed of WAIS TIQ, MMSE, verbal fluency), (3) "memory" (composed of WMS MQ, VLGT list A, list B, short- and long-term recall and recognition) and (4) "executive functioning" (composed of SDMT, Stroop interference, TMT B).

Because the time points were dependent for each participant, multilevel regression analyses (i.e. linear 

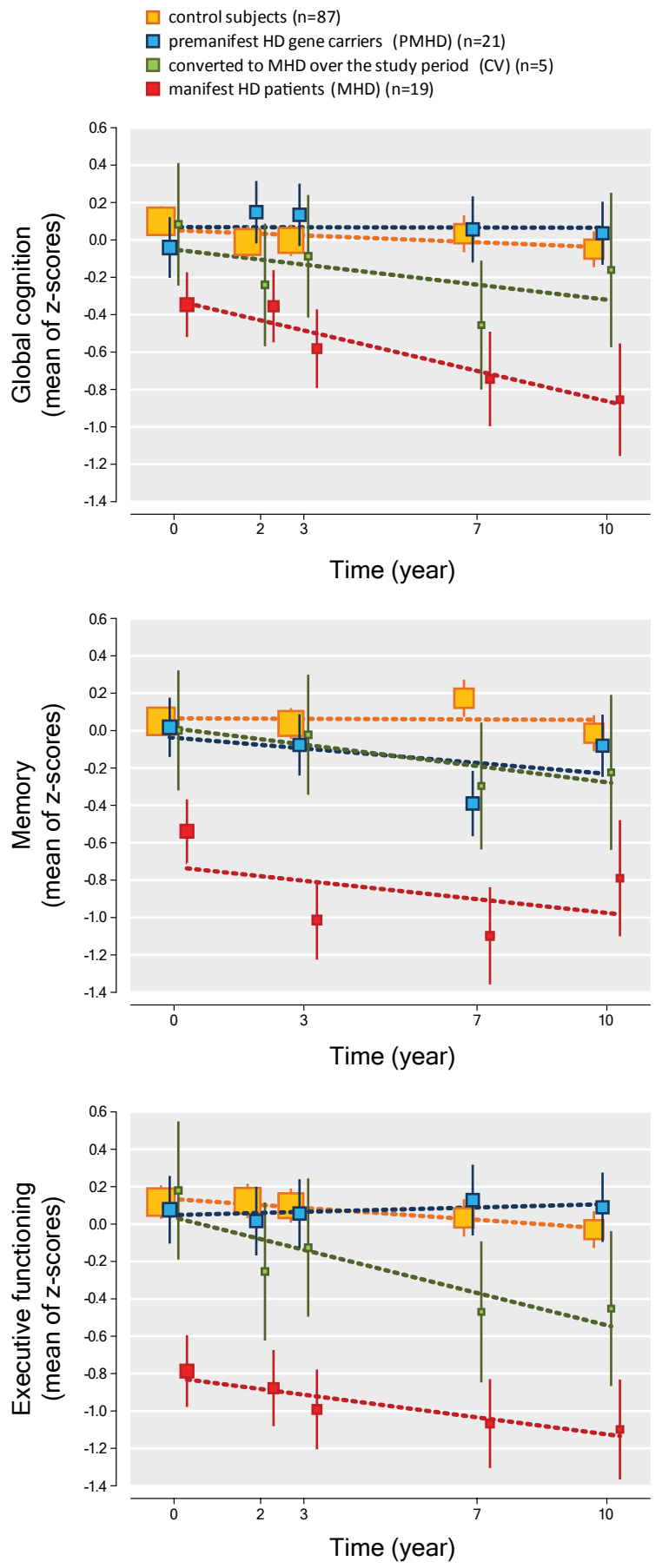

Fig. 2. Course of scores for PMHD, CV, MHD and controls on the cognitive factors over ten-years of follow-up. Note. Data are estimated marginal means (with error bars representing standard errors), adjusted for age, gender, education and motor speed.

mixed models) were used to investigate differences in baseline levels and slopes between groups on the four factors. Firstly, a crude model was constructed (model 1). Secondly, a multivariate model was constructed adjusting for age, gender, and the three categories of education (model 2). Lastly, we repeated the multilevel regression analyses for the three cognitive factors, in which we additionally adjusted for motor speed, in order to study the pure cognitive effect independent from the decline in reaction time [20] (model 3). We calculated the strength of the mediating effects of motor speed by averaging the percentage change in beta-coefficients of the significant test results between models 2 and 3. In the multilevel analyses we used a compound symmetry covariance structure consisting of up to five time points (i.e. lower level) and the subjects (i.e. higher level). The four factors were used as continuous variables, and standardized mean differences versus controls (with standard error [SE]) are presented (Fig. 2). Main analyses compared the whole premanifest HD group and the manifest HD group to controls for differences at baseline and rate of decline (i.e. slope; time* group interaction effects). Secondary analyses involved the premanifest HD group being divided into those who continued to be premanifest during the study, i.e. continual premanifest HD, and those who converted to manifest HD, i.e. converters, for comparison to controls. Additionally, the subjects whom were in the premanifest HD group at baseline were divided into 'close to onset' and 'far from onset' using a median-split of 'predicted years to onset' (median is 15.2 years), and compared to controls to test for possible influence of closeness to predicted disease onset on the cognitive factors. Post-hoc multilevel analysis on the separate cognitive tests were performed to investigate which cognitive tests are responsible for significant results in the factor analyses. Betacoefficients in the tables can be interpreted as follows: baseline differences refer to how many standard deviations an HD group differs from the controls at baseline, and slope differences refer to how many standard deviations the change in the HD groups differed from the change in the control group over the ten-year period. All tests were two-tailed with $p<0.05$ denoting statistical significance.

\section{RESULTS}

\section{Baseline demographics}

The mean $( \pm S D)$ age at baseline of the whole group of HD subjects combined was $39( \pm 11)$ years and $64 \%$ were female (Table 1). At baseline the groups were comparable for gender, educational level, total functional capacity (TFC), and depression scores. Premanifest HD subjects were younger than manifest HD 
Table 1

Baseline demographics for the groups

\begin{tabular}{|c|c|c|c|c|}
\hline & $\begin{array}{l}\text { Controls } \\
N=87\end{array}$ & $\begin{array}{l}\text { Premanifest HD } \\
\qquad N=26\end{array}$ & $\begin{array}{l}\text { Manifest HD } \\
\quad N=19\end{array}$ & $p$-value \\
\hline Age, yrs & $42(11)$ & $35(7)$ & $44(14)$ & 0.02 \\
\hline Gender, m/f & $39(45) / 48(55)$ & $7(27) / 19(73)$ & $9(47) / 10(53)$ & 0.23 \\
\hline CAG repeat length & $20(4)$ & $43(3)$ & $44(3)$ & $<0.001$ \\
\hline Higher education & $27(31)$ & $9(35)$ & $4(21)$ & 0.12 \\
\hline Depression score & $0(0 ; 1)$ & $0(0 ; 2)$ & $0(0 ; 1)$ & 0.06 \\
\hline TMS & $6(7)$ & $3(2)$ & $18(8)$ & $<0.001$ \\
\hline $\mathrm{TFC}$ & $13(13 ; 13)$ & $13(13 ; 13)$ & $13(13 ; 13)$ & 0.06 \\
\hline Disease burden $^{a}$ & & $259(89)$ & $332(79)$ & 0.008 \\
\hline Expected until onset ${ }^{\mathrm{b}}$, yrs & & $16(8)$ & & \\
\hline
\end{tabular}

Note: Data are mean (SD) for continuous variables, number (\%) for gender and education and median (interquartile range) for TFC and depression score. ANOVA was used for comparisons of age, CAG and TMS variables $\chi^{2}$-tests for gender and education variables and Kruskal-Wallis tests for depression score and total functional capacity variable. $\mathrm{TFC}=$ total functional capacity, TMS = total motor score ${ }^{\text {a }}$ Based on formula '(CAG-35.5)*age)' by Penney et al. (1997). ${ }^{b}$ Based on survival analysis formula by Langbehn et al. (2004).

$(p=0.03)$ and control $(p=0.04)$ subjects. The manifest HD group had a higher TMS than the controls $(p<0.001)$. The burden of the manifest HD subjects was higher compared to the premanifest HD subjects $(p=0.008)$. Raw cognitive scores are given in Supplementary Table 1.

The subjects who completed the ten-year follow-up $(n=68)$ were comparable to the drop-outs $(n=64)$ on age at baseline $(p=0.92)$, gender $(p=0.48)$ and education $(p=0.12)$. The drop-outs showed lower TFC $(p=0.006)$ and higher TMS scores $(p=0.02)$ (data not shown).

\section{Baseline differences and change over time for premanifest $H D$ and manifest $H D$ on the four factors}

The baseline differences and change over the tenyear follow-up period for the premanifest HD and manifest HD groups compared with the controls are presented in Table 2. The analyses adjusted for age, gender and education showed lower baseline scores for the manifest HD group on the motor speed factor (Table 2B). The scores were on average $0.72 \mathrm{SD}$ $(\mathrm{SE}=0.22 ; p=0.002)$ lower than the scores of the controls. For the executive functioning and memory factors the same pattern was found; for executive functioning factor the scores were 0.79 $\mathrm{SD}(\mathrm{SE}=0.17$; $p<0.001)$ lower and for the memory factor $0.61 \mathrm{SD}$ $(\mathrm{SE}=0.17 ; p<0.001)$ lower. Only for the latter factor both HD groups showed a difference in change over time compared to that of controls. The manifest HD group deteriorated with on average $0.61 \mathrm{SD}(\mathrm{SE}=0.24$; $p=0.01$ ) over ten-year follow-up. The premanifest HD group deteriorated with $0.32 \mathrm{SD}(\mathrm{SE}=0.15 ; p=0.04)$.
Post-hoc analysis to investigate which individual cognitive tests caused significant factor results revealed that for the memory factor both short and long term memory on the VLGT and the WMS MQ showed lower baseline scores for the manifest subjects compared to the controls (SD between 0.79 and 0.69, $p$-values between 0.001 and $<0.001$ ) (Supplementary Table 2A). Concerning change over time the premanifest subjects deteriorated on long term recognition of the VLGT (0.48 SD [SE=0.23, $p=0.04]$ ), and the manifest subjects deteriorated in the WMS MQ (0.70 SD [SE $=0.23 ; p=0.003])$. The significant baseline difference on the executive functioning factor was driven by worse baseline scores for the SDMT $(0.85 \mathrm{SD}[\mathrm{SE}=0.20 ; p<0.001])$ and TMT-B $(0.77 \mathrm{SD}$ $[\mathrm{SE}=0.20 ; p<0.001])$.

To partition out the influence of motor functioning on cognition motor speed was added as an additional covariate to the multilevel regression analyses on the cognitive factors (Table 2C). Now, only the manifest HD group showed lower baseline scores compared to controls on the executive functioning $(-0.73 \mathrm{SD}(\mathrm{SE}=0.17) ; p<0.001)$ and memory $(-0.62$ $(\mathrm{SE}=0.17) ; p<0.001)$ factors. The adjustment for motor speed resulted in a reduction in size of the statistically significant beta-coefficients by on average $20.2 \%$. This indicates a substantial mediating effect of the reduction in motor speed over time on the cognitive factors.

Supplementary Table 2B shows the data of the individual cognitive tests adjusted for age, gender, education and the motor factor. Here, post-hoc analyses revealed that the memory factor was driven by significant baseline differences for the manifest group compared to the controls on both the direct and the 
Table 2

Changes over ten-year follow-up on cognitive and motor factors for premanifest and manifest HD compared to controls

\begin{tabular}{|c|c|c|c|c|}
\hline & $\begin{array}{c}\text { Controls } \\
N=87\end{array}$ & $\begin{array}{l}\text { Premanifest HD } \\
\qquad N=26\end{array}$ & $\begin{array}{c}\text { Manifest HD } \\
\qquad N=19\end{array}$ & $P$-value \\
\hline \multicolumn{5}{|l|}{ 2A. Crude } \\
\hline \multicolumn{5}{|l|}{ Motor } \\
\hline - Baseline difference & Ref. & $-0.16($ SE 0.20$)$ & $-0.80(\mathrm{SE} 0.24)^{* *}$ & 0.004 \\
\hline - Slope difference & Ref. & $-0.13($ SE 0.18$)$ & $0.27(\mathrm{SE} 0.34)$ & 0.51 \\
\hline \multicolumn{5}{|l|}{ Global Cognition } \\
\hline - Baseline difference & Ref. & $0.00(\mathrm{SE} 0.15)$ & $-0.39(\mathrm{SE} 0.17)$ & 0.08 \\
\hline - Slope difference & Ref. & $-0.03(\mathrm{SE} 0.13)$ & $-0.49(\mathrm{SE} 0.21)$ & 0.07 \\
\hline \multicolumn{5}{|l|}{ Executive functioning } \\
\hline - Baseline difference & Ref. & $-0.12($ SE 0.18$)$ & $-0.95(\mathrm{SE} 0.20)^{* * *}$ & $<0.001$ \\
\hline - Slope difference & Ref. & $0.12(\mathrm{SE} 0.10)$ & $-0.26($ SE 0.16$)$ & 0.08 \\
\hline \multicolumn{5}{|l|}{ Memory } \\
\hline - Baseline difference & Ref. & $-0.03(\mathrm{SE} 0.15)$ & $-0.68(\mathrm{SE} 0.18)^{* * *}$ & 0.001 \\
\hline - Slope difference & Ref. & $-0.34(\mathrm{SE} 0.15)^{*}$ & $-0.60(\mathrm{SE} 0.24)^{*}$ & 0.01 \\
\hline \multicolumn{5}{|c|}{ 2B. Adjusted - age, gender, education } \\
\hline \multicolumn{5}{|c|}{ Motor } \\
\hline - Baseline difference & Ref. & $-0.08($ SE 0.20$)$ & $-0.72(\mathrm{SE} 0.22)^{* *}$ & 0.007 \\
\hline - Slope difference & Ref. & $-0.13($ SE 0.18$)$ & 0.25 (SE 0.34) & 0.55 \\
\hline \multicolumn{5}{|l|}{ Global Cognition } \\
\hline - Baseline difference & Ref. & 0.04 (SE 0.14) & $-0.32(\mathrm{SE} 0.16)$ & 0.12 \\
\hline - Slope difference & Ref. & $-0.03(\mathrm{SE} 0.13)$ & $-0.51(\mathrm{SE} 0.21)$ & 0.05 \\
\hline \multicolumn{5}{|l|}{ Executive functioning } \\
\hline - Baseline difference & Ref. & -0.27 (SE 0.16) & $-0.79(\text { SE } 0.17)^{* * *}$ & $<0.001$ \\
\hline - Slope difference & Ref. & 0.11 (SE 0.10) & $-0.28($ SE 0.16$)$ & 0.08 \\
\hline \multicolumn{5}{|l|}{ Memory } \\
\hline - Baseline difference & Ref. & $-0.08(\mathrm{SE} 0.15)$ & $-0.61(\mathrm{SE} 0.17)^{* * *}$ & 0.002 \\
\hline - Slope difference & Ref. & $-0.32(\operatorname{SE~} 0.15)^{*}$ & $-0.61(\mathrm{SE} 0.24)^{*}$ & 0.007 \\
\hline \multicolumn{5}{|c|}{$\begin{array}{l}2 \text { C. Adjusted - age, gender, education, motor speed } \\
\text { Global Cognition }\end{array}$} \\
\hline - Baseline difference & Ref. & 0.03 (SE 0.14) & $-0.28($ SE 0.16$)$ & 0.21 \\
\hline - Slope difference & Ref. & $0.06(\mathrm{SE} 0.14)$ & $-0.49(\mathrm{SE} 0.25)$ & 0.11 \\
\hline \multicolumn{5}{|l|}{ Executive functioning } \\
\hline - Baseline difference & Ref. & $-0.25(\mathrm{SE} 0.15)$ & $-0.73(\mathrm{SE} 0.17)^{* * *}$ & $<0.001$ \\
\hline - Slope difference & Ref. & $0.11(\mathrm{SE} 0.10)$ & $-0.21(\mathrm{SE} 0.19)$ & 0.24 \\
\hline \multicolumn{5}{|l|}{ Memory } \\
\hline - Baseline difference & Ref. & $-0.12($ SE 0.15$)$ & $-0.62(\mathrm{SE} 0.17)^{* * *}$ & 0.002 \\
\hline - Slope difference & Ref. & $-0.17($ SE 0.15) & -0.44 (SE 0.28) & 0.20 \\
\hline
\end{tabular}

Note: Data are standardized mean differences versus controls (standard errors). In all multilevel regression analyses a compound symmetry covariance matrix (CS) was used. ${ }^{*} p<0.05,{ }^{* *}<0.01,{ }^{* * *}<0.001$ indicate significant differences compared to the control group in post-hoc tests.

short and long term recall of the VLGT (SD between 0.76 and 1.08; $p$-values between 0.01 and $<0.001$ ) and the WMS MQ (0.75 SD [SE $=0.21 ; p<0.001)$. The executive functioning factor was driven by worse baseline scores for the manifest group on both the SDMT $(0.82 \mathrm{SD}[\mathrm{SE}=0.20 ; p<0.001])$ and the TMT-B $(0.74$ $\mathrm{SD}[\mathrm{SE}=0.21 ; p<0.001])$.

Baseline differences and change over time for continual premanifest $H D$ and converters on the four factors

Next we analysed converters $(n=5)$ separated from the continual subjects premanifest HD $(n=21)$. The baseline and slope data for the analysis with the con- verters separated from the original premanifest $\mathrm{HD}$ group are shown in Table 3A. Figure 2 depicts data for all four groups. The analysis adjusted for age, gender and education showed a deterioration over time on the executive functioning factor for the converter group ( $-0.59 \mathrm{SD}(\mathrm{SE}=0.19) ; p=0.002)$ as compared to controls, while the continual premanifest HD group improved with on average $0.27 \mathrm{SD}(\mathrm{SE}=0.10$; $p=0.01$ ) compared to controls. The converters group also deteriorated over time on the memory factor, with $0.83 \mathrm{SD}(\mathrm{SE}=0.30 ; p=0.006)$.

Post-hoc analysis on the individual cognitive tests adjusted for age, gender and education (Supplementary Table 3A) showed that the significant slope difference for memory factor was caused by significant deteriora- 
Table 3

Changes over ten-year follow-up on cognitive and motor factors for premanifest subgroups compared to controls

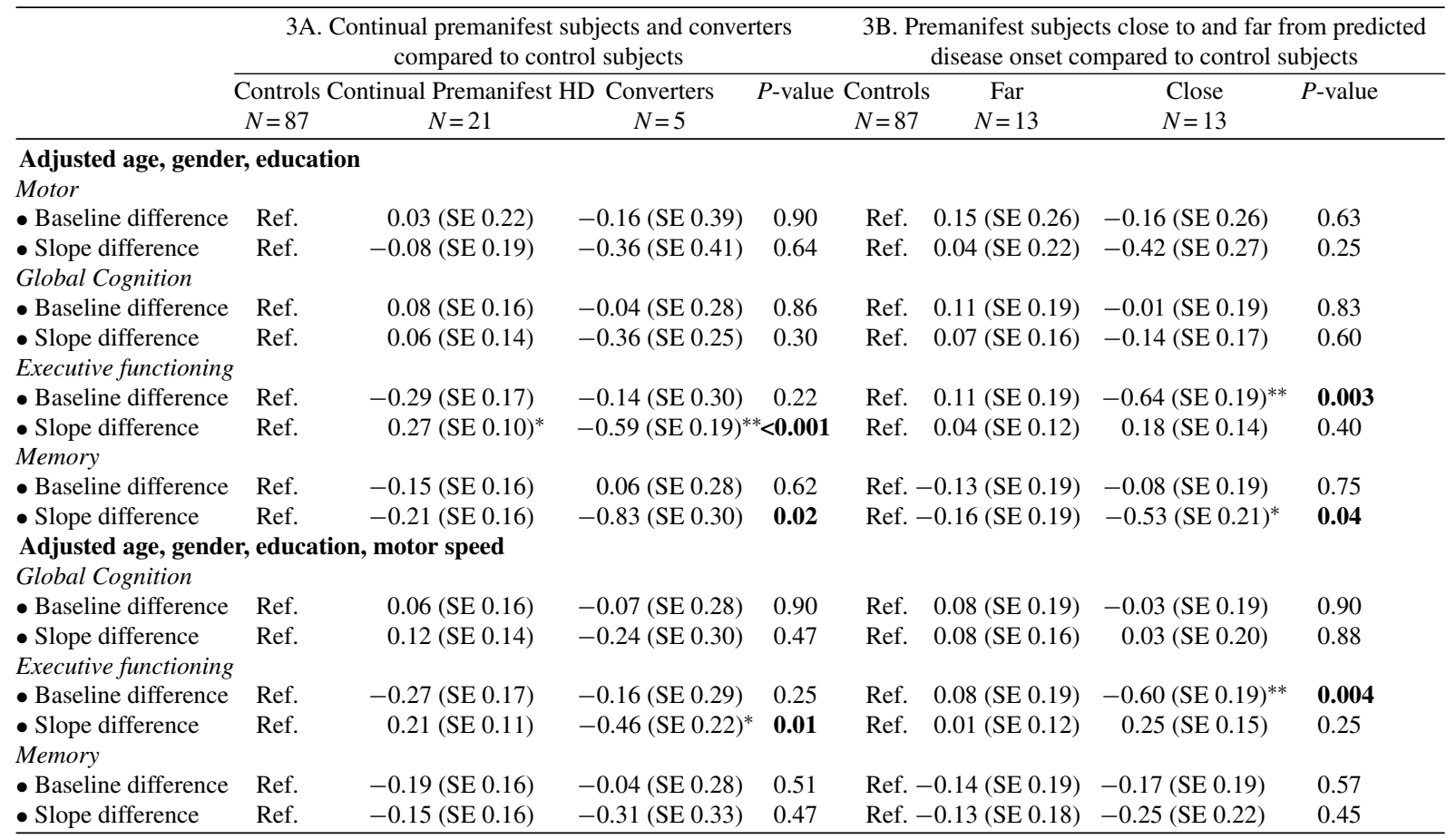

Note: Data are standardized mean differences versus controls (standard errors). In all multilevel regression analyses a compound symmetry covariance matrix (CS) was used. ${ }^{*} p<0.05,{ }^{* *}<0.01,{ }^{* * *}<0.001$ indicate significant differences compared to the control group in post-hoc tests.

tion for the converter group compared to the controls on the WMS MQ of $0.88 \mathrm{SD}(\mathrm{SE}=0.27 ; p=0.001)$. The executive functioning factor was driven by the SDMT, where the converters deteriorated with on average 0.70 $\mathrm{SD}(\mathrm{SE}=0.26, p=0.008)$.

With the introduction of motor speed as additional covariate the deterioration for the converter group, now $0.46 \mathrm{SD}$ ( $\mathrm{SE}=0.22, p=0.04$ ), remained (Supplementary Table $3 \mathrm{~B})$. The improvement of the continual premanifest HD group of now $0.21 \mathrm{SD}(\mathrm{SE}=0.11$, $p=0.052$ ) was only borderline significant. Post-hoc analyses on component tests of the executive functioning factor revealed that the deterioration for the converter group was solely caused by lower scores for this group on the SDMT, of on average 0.64 SD $(\mathrm{SE}=0.30, p=0.04)$.

\section{Baseline differences and change over time for subjects close to and far from predicted disease onset compared controls on the four factors}

Table 3B presents the baseline and slope differences of the motor speed and cognitive factors for the premanifest HD group divided into close to and far from predicted disease onset. For the executive functioning factor, analysis adjusted for age, gender and education showed lower baseline scores for the close to onset group of $0.64 \mathrm{SD}(\mathrm{SE}=0.19 ; p=0.001)$ compared to controls. The 'close' group also showed a deterioration over the ten-year follow-up of $0.53 \mathrm{SD}(\mathrm{SE}=0.21$; $p=0.01$ ) on the memory factor compared to controls. Investigation of the individual cognitive tests making up the factors revealed that the significant slope difference for the memory factor was based on the 'close' group deteriorating with on average 0.61 SD $(\mathrm{SE}=0.19) ; p=0.001)$ compared to controls over the ten-year follow-up (Supplementary Table 4A). Significant baseline differences on the executive functioning factor were the result of lower baseline scores of 0.77 $\mathrm{SD}(\mathrm{SE}=0.23 ; p=0.001)$ for the 'close' group compared to the control group on the SDMT.

When motor speed was added to the analyses on the cognitive factors results showed that lower baseline scores for the close to onset group on the executive functioning factor persisted. The scores were on average $0.60 \mathrm{SD}(\mathrm{SE}=0.19 ; p=0.002)$ lower as compared to control subjects. Again, this result was mainly based on the 'close' group scoring 0.73 SD ( $\mathrm{SE}=0.22$; $p=0.001$ ) lower on the SDMT compared to the controls (Supplementary Table 4B). 


\section{DISCUSSION}

The present study reports on ten-year follow-up of cognitive functioning in premanifest and manifest HD. We found that manifest HD subjects scored significantly worse than controls on the factors of motor, executive functioning and memory. Over the ten-year follow-up they also showed deterioration on the memory factor, while change over time on the other factors was comparable to that of controls. Additionally, the premanifest subjects who converted to manifest disease were the only group to show significant deterioration over the ten-year follow-up period compared to the control subjects on the executive functioning factor and to lesser extent on the memory factor, whereas the premanifest participants who remained without clinical diagnosis showed no such change.

That the manifest HD group had lower mean baseline scores on the motor speed, executive and memory factors is not surprising as deficits in these areas of functioning are well established $[21,22]$. Longitudinally, this group showed deterioration on the memory factor, which is in line with other longitudinal studies on cognition in manifest HD [15, 23]. When we controlled for motor functioning all factors ceased to show deterioration for this group. That in our fully adjusted model the deterioration on all cognitive factors did not differ from that of the control group seems counterintuitive, due to the neurodegenerative nature and the known cognitive deficits in manifest HD [24]. The most likely explanation is the selective attrition that takes place in longitudinal studies, where the subjects most vulnerable to cognitive decline have a larger chance of becoming lost to follow-up. Indeed, in our manifest HD group 14 out of the initial 19 were lost to follow-up, which likely resulted in a pronounced underestimation of the cognitive decline in this group.

The cognitive course over time for the subjects who were premanifest at baseline was most remarkable. Those who converted to manifest disease showed deterioration on both the memory and the executive functioning factors, as compared to controls. The decline on the executive factor was however most pronounced, a decline that was even greater than that of the manifest subjects. These results are supported by the findings of Solomon et al. [14] who also performed a ten-year follow-up study. They used three cognitive tasks (i.e. subtests from the WAIS-R) and six motor and psychomotor tasks and found that their converter group showed greater rates of decline on the motor and psychomotor tasks compared with non-converters. Similarly, Rupp et al. [25] also reported faster deteri- oration for their converter group, especially in tests of psychomotor abilities.

In contrast, the subjects in our study who remained premanifest over the ten year follow-up showed no deterioration on any of the cognitive factors or the individual cognitive tests. They even showed a slight improvement over time on the executive functioning factor compared to controls, which was lessened to borderline significant when we introduced the motor speed factor to the analysis. This result could reflect a biphasic or non-linear pattern of progress of executive functioning, where premanifest subjects are able to maintain test requirements to the same, or even better, extent as controls, but where a rapid decline occurs close to or directly after disease onset. Non-linear progression of premanifest test performance has also been suggested by other authors. Snowden et al. [12] and Paulsen et al. [26] found that decline on certain cognitive measures (i.c. memory and executive functioning) seems to accelerate close clinical disease onset. Rupp and colleagues also observed an improvement for their premanifest group, which was far from predicted disease onset [25]. Rupp et al. attributed this to learning effects, however, this is a less likely explanation for our premanifest group as no significant improvement was found on the other factors. However, the influence of practice effects cannot be ruled out, especially since alternative versions were only available and used for certain, but not all, tests (i.c. SDMT and TMT). As Duff et al. have demonstrated [27], the influence of practice effects in repeated testing should be considered as a clarification for our results. Another explanation could again be the selective drop-out of subjects who are cognitively and functionally already impaired, even though only 4 of the original premanifest subjects were lost to follow-up. Overall, our results indicate that tests of memory, and to a somewhat larger extent, executive functioning are likely to be most sensitive to subtle deteriorations during the premanifest and transition phases of HD.

Splitting the original premanifest group (including those who converted to manifest HD over time) into 'far' (more than 15.2 years from predicted disease onset) and 'close' (15.2 or less years to onset) strengthened our hypothesis of non-linear progression for the memory and executive functioning factors. We found worse performance for the close group compared to the control group. This finding implicates that cognitive decline, and more specifically executive dysfunctioning and memory decline, is present and can be detected even before overt (motor-symptomatic) disease onset, an assumption that is also shared by other authors [28]. 
Our findings strengthen the idea proposed by Papp et al. [11] amongst others, that premanifest subjects should not be regarded as one group, but that at least two different subgroups (i.e. close to and far from predicted disease onset) exist that indeed demonstrate different cognitive profiles. Further analyses revealed that poorer performance on the SDMT contributed most to executive dysfunction. This finding corroborates other findings where the SDMT proved most sensitive to the earliest cognitive change in premanifest HD [23, $29,30]$. Our results suggest that the SDMT is a sensitive instrument to track disease progression over time, especially around clinical onset.

Besides the progression of cognitive factors over a ten-year period we were also interested in the effect that motor functioning has on cognition, especially in the premanifest phase of HD. As for most cognitive tests some kind of motoric response (e.g. writing, drawing, speaking) is required, and as subtle motor disturbances have already been found in premanifest groups [20, 31], this effect is worth investigating. We approached this by using the test that we hypothesized to be mainly motoric and relatively free of cognitive effort; the simple reaction time (RT) test (i.e. responding as quickly as possible to a tone or a light in two separate conditions by pushing a response button) as a covariate in our analyses. We found evidence for substantial mediating effects of motor speed on all three cognitive factors. When we adjusted for motor speed, results on particularly the global cognition and memory factors ceased to be significant. Also, $p$-values became less significant. That differences weaken and disappear with the introduction of a motor measure, underlines the importance of taking this motor influence into account while investigating cognition in HD. Studies that do not take into account the influence of motor function in the HD groups could potentially result in an overestimation of the 'pure' cognitive effect.

This is one of the few cognitive studies where the effect of motor functioning on cognitive test scores is quantitatively studied. However, we acknowledge that the choice for motor speed was an arbitrary choice and that we did not a-priori investigate whether this variable indeed (only) measures motor speed. There is a possibility that this variable also measures some kind of cognitive construct as our memory results were also affected by the introduction of the motor speed variable. Memory tasks are often less dependent on motor abilities than executive tasks, as they often only require a verbal response. On the other hand, from our supplementary data on the individual cognitive tests we can see that the WMS MQ is especially affected by the introduction of the motor speed variable. One of the sub-tests making up the WMS MQ is visual reproduction, where the patients is required to draw from memory, and this element could be especially sensitive to motor speed influence. The other memory tests, e.g. direct and long term recall from the VLGT, seem to be less affected by the introduction of the motor variable, strengthening our hypothesis that we are controlling for motor functioning. Nonetheless, future research is needed to more thoroughly investigate the potential influence of motor functioning on cognitive testing.

However, we feel that our attempt to quantify the influence of motor functioning on cognitive scores has provided some interesting insights on differential influences on cognitive measures in HD, even though our results should be interpreted with caution. If our hypothesis that our motor factor measures pure motor speed is correct, our results indicate that commonly used tests for global functioning and memory used in $\mathrm{HD}$ are sensitive for the progressive motor disturbances characteristic for $\mathrm{HD}$, which reduces their ability to pick up cognitive decline. It is unlikely that no decline on these measures occurs in the premanifest phase, as memory decline, and to a lesser extent also global functioning decline, have consistently been found in premanifest HD gene carriers [24, 32,33 ]. Indeed, without the correction for motor speed, both converters and subjects close to predicted disease onset also showed deterioration compared to controls on the memory factor. Our findings suggest however, that these results could have been mediated by motor functioning and are likely to be smaller in extend. Nonetheless, tests of executive functioning, and the SDMT in particular, do show sensitivity for motor disturbances, but remain sensitive enough to pick up cognitive decline, which makes them best suited for use in longitudinal follow-up studies and clinical trials.

Our study adds to the existing literature as one of the longest follow-up studies on cognition in both manifest and premanifest HD. The diverse battery of cognitive tests is also a strong aspect of this study. Moreover, adjusting for the influence of motor speed has provided insights about the negative impact of motor functioning on cognition. However, some limitations have to be mentioned. During the study half of the initial participants were lost to follow-up, introducing a potential attrition bias. Therefore, results of both manifest subjects and converters should be interpreted with caution as the numbers in these groups were small and variability in test scores is substantial. Additionally, the influence of medication use (e.g. neuroleptics) was not investigated in this study, even though the effect 
can be both beneficial and disadvantageous. To avoid overestimation of results, future studies are advised to administer a motor task alongside cognitive tasks, to be able to disentangle the influence of motor disturbances on cognitive functioning in HD.

We conclude that both memory and executive functioning are sensitive in picking up premanifest decline in subjects close to their predicted disease onset. The latter cognitive domain is specifically sensitive, with the SDMT proven to be the most sensitive individual test. Our results imply that the course of executive functioning in the premanifest stage is not uniform and that in subjects furthest away from disease onset changes are difficult to detect. Additionally, we have found evidence for influence of motor speed on cognitive outcome measures.

\section{ACKNOWLEDGMENTS}

We thank M.-N. Witjes-Ané, MSc, PhD, C.K. Jurgens, MSc, PhD, J.P.P. van Vugt, MD, PhD, Y.A.M. Grimbergen, MD, PhD and S.J.A. van den Bogaard, $\mathrm{MD}, \mathrm{PhD}$, for their help in data collection.

\section{FUNDING AND FINANCIAL DISCLOSURES}

This research was not funded. Raymund Roos, Ellen Hart and Eve Dumas report that their institution receives payment from the European Huntington's Disease Network for the Registry and Track-HD projects. Erik Giltay and Huub Middelkoop report no disclosures.

\section{CONFLICT OF INTEREST}

The authors have no conflict of interest to report.

\section{SUPPLEMENTARY MATERIAL}

Supplementary material can be found here: http:// dx.doi.org/10.3233/JHD-130059

Supplementary Table 1 . Raw cognitive test scores at baseline and ten-year follow-up

Supplementary Table 2. Changes over ten-year follow-up on cognitive tests for premanifest HD and manifest HD subjects compared to controls.

Supplementary Table 3. Changes over ten-year follow-up on cognitive tests for continual premanifest HD and converter subjects compared to controls.

Supplementary Table 4. Changes over ten-year follow-up on cognitive tests for premanifest HD sub- jects far from and close to predicted onset compared to controls.

\section{REFERENCES}

[1] Roos RA. Huntington's disease: A clinical review. Orphanet J Rare Dis. 2010;5:40

[2] Guidelines for the molecular genetics predictive test in Huntington's disease. International Huntington Association (IHA) and the World Federation of Neurology (WFN) Research Group on Huntington's Chorea. Neurology. 1994;44:1533-6.

[3] HDCRG. A novel gene containing a trinucleotide repeat that is expanded and unstable on Huntington's disease chromosomes. The Huntington's Disease Collaborative Research Group. Cell. 1993;72:971-83.

[4] Stout JC, Weaver M, Solomon AC, Queller S, Hui S, Johnson SA, Gray J, Beristain X, Wojcieszek J, Foroud T. Are cognitive changes progressive in prediagnostic HD? Cogn Behav Neurol. 2007;20:212-8.

[5] Novak MJ, Warren JD, Henley SM, Draganski B, Frackowiak RS, Tabrizi SJ. Altered brain mechanisms of emotion processing in pre-manifest Huntington's disease. Brain. 2012;135:1165-79.

[6] Peavy GM, Jacobson MW, Goldstein JL, Hamilton JM, Kane A, Gamst AC, Lessig SL, Lee JC, Corey-Bloom J. Cognitive and functional decline in Huntington's disease: Dementia criteria revisited. Mov Disord. 2010;25:1163-69.

[7] Verny C, Allain P, Prudean A, Malinge MC, Gohier B, Scherer C, Bonneau D, Dubas F, Le GD. Cognitive changes in asymptomatic carriers of the Huntington disease mutation gene. Eur J Neurol. 2007;14:1344-50.

[8] Witjes-Ane MN, Vegter-van der Vlis M, van Vugt JP, Lanser JB, Hermans J, Zwinderman AH, van Ommen GJ, Roos RA. Cognitive and motor functioning in gene carriers for Huntington's disease: A baseline study. J Neuropsychiatry Clin Neurosci. 2003;15:7-16.

[9] Soliveri P, Monza D, Piacentini S, Paridi D, Nespolo C, Gellera C, Mariotti C, Albanese A, Girotti F. Cognitive and psychiatric characterization of patients with Huntington's disease and their at-risk relatives. Neurol Sci. 2002;23(Suppl 2):S105-S6.

[10] Paulsen JS. Cognitive impairment in Huntington disease: Diagnosis and treatment. Curr Neurol Neurosci Rep. 2011;11:474-83.

[11] Papp KV, Kaplan RF, Snyder PJ. Biological markers of cognition in prodromal Huntington's disease: A review. Brain Cogn. 2011;77:280-91.

[12] Snowden JS, Craufurd D, Thompson J, Neary D. Psychomotor, executive, and memory function in preclinical Huntington's disease. J Clin Exp Neuropsychol. 2002;24:13345.

[13] Brandt J, Inscore AB, Ward J, Shpritz B, Rosenblatt A, Margolis RL, Ross CA. Neuropsychological deficits in Huntington's disease gene carriers and correlates of early "conversion". J Neuropsychiatry Clin Neurosci. 2008;20:466-72.

[14] Solomon AC, Stout JC, Weaver M, Queller S, Tomusk A, Whitlock KB, Hui SL, Marshall J, Jackson JG, Siemers ER, Beristain X, Wojcieszek J, Foroud T. Ten-year rate of longitudinal change in neurocognitive and motor function in prediagnosis Huntington disease. Mov Disord. 2008;23:18306.

[15] Hart E, Middelkoop H, Jurgens CK, Witjes-Ane MN, Roos RA. Seven-year clinical follow-up of premanifest carriers of Huntington's disease. PLoS Curr. 2011;3:RRN1288. 
[16] Witjes-Ane MN, Mertens B, van Vugt JP, Bachoud-Levi AC, van Ommen GJ, Roos RA. Longitudinal evaluation of "presymptomatic" carriers of Huntington's disease. J Neuropsychiatry Clin Neurosci. 2007;19:310-7.

[17] Huntington Study Group. Unified Huntington's Disease Rating Scale: Reliability and consistency. Huntington Study Group. Mov Disord. 1996;11:136-42.

[18] Tabrizi SJ, Langbehn DR, Leavitt BR, Roos RA, Durr A, Craufurd D, Kennard C, Hicks SL, Fox NC, Scahill RI, Borowsky B, Tobin AJ, Rosas HD, Johnson H, Reilmann R, Landwehrmeyer B, Stout JC. Biological and clinical manifestations of Huntington's disease in the longitudinal TRACK-HD study: Cross-sectional analysis of baseline data Lancet Neurol. 2009;8:791-801.

[19] Langbehn DR, Brinkman RR, Falush D, Paulsen JS, Hayden MR. A new model for prediction of the age of onset and penetrance for Huntington's disease based on CAG length. Clin Genet. 2004:65:267-77.

[20] Biglan KM, Ross CA, Langbehn DR, Aylward EH, Stout JC, Queller S, Carlozzi NE, Duff K, Beglinger LJ, Paulsen JS. Motor abnormalities in premanifest persons with Huntington's disease: The PREDICT-HD study. Mov Disord. 2009;24:1763-72.

[21] Beglinger LJ, Duff K, Allison J, Theriault D, O'Rourke JJ, Leserman A, Paulsen JS. Cognitive change in patients with Huntington disease on the Repeatable Battery for the Assessment of Neuropsychological Status. J Clin Exp Neuropsychol. 2010;32:573-8

[22] Novak MJ, Tabrizi SJ. Huntington's disease: Clinical presentation and treatment. Int Rev Neurobiol. 2011;98:297323.

[23] Lemiere J, Decruyenaere M, Evers-Kiebooms G, Vandenbussche E, Dom R. Cognitive changes in patients with Huntington's disease (HD) and asymptomatic carriers of the HD mutation-a longitudinal follow-up study. J Neurol. 2004;251:935-42.

[24] Dumas EM, van den Bogaard SJ, Middelkoop HA, Roos RA A review of cognition in Huntington's disease. Front Biosci (Schol Ed). 2013;5:1-18.

[25] Rupp J, Blekher T, Jackson J, Beristain X, Marshall J, Hui S, Wojcieszek J, Foroud T. Progression in prediagnostic Huntington disease. J Neurol Neurosurg Psychiatry. 2010;81:379-84
[26] Paulsen JS, Langbehn DR, Stout JC, Aylward E, Ross CA, Nance M, Guttman M, Johnson S, MacDonald M, Beglinger LJ, Duff K, Kayson E, Biglan K, Shoulson I, Oakes D, Hayden M. Detection of Huntington's disease decades before diagnosis: The Predict-HD study. J Neurol Neurosurg Psychiatry. 2008;79:874-80.

[27] Duff K, Beglinger LJ, Schultz SK, Moser DJ, McCaffrey RJ, Haase RF, Westervelt HJ, Langbehn DR, Paulsen JS. Practice effects in the prediction of long-term cognitive outcome in three patient samples: A novel prognostic index. Arch Clin Neuropsychol. 2007;22:15-24.

[28] Papp KV, Snyder PJ, Mills JA, Duff K, Westervelt HJ, Long JD, Lourens S, Paulsen JS. Measuring Executive Dysfunction Longitudinally and in Relation to Genetic Burden, Brain Volumetrics, and Depression in Prodromal Huntington Disease. Arch Clin Neuropsychol. 2012.

[29] Paulsen JS, Zhao H, Stout JC, Brinkman RR, Guttman M, Ross CA, Como P, Manning C, Hayden MR, Shoulson I. Clinical markers of early disease in persons near onset of Huntington's disease. Neurology. 2001;57:658-62.

[30] Stout JC, Jones R, Labuschagne I, O'Regan AM, Say MJ, Dumas EM, Queller S, Justo D, Santos RD, Coleman A, Hart EP, Durr A, Leavitt BR, Roos RA, Langbehn DR, Tabrizi SJ, Frost C. Evaluation of longitudinal 12 and 24 month cognitive outcomes in premanifest and early Huntington's disease. J Neurol Neurosurg Psychiatry. 2012;83:687-94.

[31] Kirkwood SC, Siemers E, Stout JC, Hodes ME, Conneally PM, Christian JC, Foroud T. Longitudinal cognitive and motor changes among presymptomatic Huntington disease gene carriers. Arch Neurol. 1999;56:563-8.

[32] Stout JC, Paulsen JS, Queller S, Solomon AC, Whitlock KB, Campbell JC, Carlozzi N, Duff K, Beglinger LJ, Langbehn DR, Johnson SA, Biglan KM, Aylward EH. Neurocognitive signs in prodromal Huntington disease. Neuropsychology. 2011;25:1-14.

[33] Robins Wahlin TB, Larsson MU, Luszcz MA, Byrne GJ. WAIS-R features of preclinical Huntington's disease: Implications for early detection. Dement Geriatr Cogn Disord. 2010;29:342-50 